\title{
TRK inhibitors: toward an era of agnostic targeted therapies in oncology
}

\author{
Khalil Saleh1, Nadine Khalifeh-Saleh \& Hampig Raphael Kourie*,1 \\ ${ }^{1}$ Hematology-Oncology Department, Faculty of Medicine, Saint Joseph University of Beirut, Beirut, Lebanon \\ *Author for correspondence: hampig.kourie@hotmail.com \\ "The aim of this paper is to define the function of NTRK genes, to resume the role of NTRK fusions \\ and carcinogenesis, the detection of NTRK fusions and the available data concerning NTRK \\ inhibitors."
}

First draft submitted: 26 April 2019; Accepted for publication: 2 July 2019; Published online: 5 September 2019

Keywords: entrectinib • larotrectinib $\bullet$ NTRK fusion $\bullet$ TRK inhibitors $\bullet$ tumor agnostic

Traditionally, the majority of cancer therapies were used to treat specific organ or tissue tumors such as prostate cancer, breast cancer or lung cancer. However, in the last few years, tumor-agnostic treatment has emerged. It is defined as a drug used to treat any kind of cancer regardless of tissue of origin and when the tumor shows specific molecular alterations which can be targeted by the therapy or which predict that the treatment might be efficacious. Microsatellite instability-high (MSI-H) or mismatch repair deficient (dMMR) were the first reported agnostic biomarkers. Pembrolizumab, a PD-1 inhibitor was the first tumor-agnostic treatment approved by the US FDA in May 2017. It has been approved in the treatment of pediatric and adults patients with unresectable or metastatic solid tumors which present MSI-H or dMMR as a biomarker [1]. Similarly, in August 2017, the FDA approved nivolumab, another PD-1 inhibitor, in the treatment of MSI-H or dMMR metastatic colorectal cancer that has progressed following treatment [2]. More recently, larotrectinib, a pan-TRK inhibitor is approved by the FDA in November 2018 for the treatment of pediatric and adult tumors that have a NTRK gene fusion [3]. Larotrectinib is the first tumor-agnostic-targeted therapy developed and approved in oncology. The aim of this paper is to define the function of NTRK genes, to resume the role of NTRK fusions and carcinogenesis, the detection of NTRK fusions and the available data concerning NTRK inhibitors.

\section{Function of NTRK genes}

The NTRK genes NTRK1, NTRK2 and NTRK3 encode the receptor tyrosine kinases, TRKA, TRKB and TRKC, respectively. The TRKs are transmembrane receptors composed of extracellular ligand-binding domain, transmembrane domain and intracellular ATP-binding Ig2 [4]. These receptors are activated by binding with high affinity to their ligands: neurotrophin nerve growth factor for TRKA, brain-derived neurotrophic factor or neurotrophin 4 for TRKB and neurotrophin 3 for TRKC. This activation leads to receptor dimerization and autophosphorylation of intracellular tyrosine kinase residues driving for downstream signaling and promoting cell proliferation, differentiation and survival [5]. TRKs play a crucial role in the neuronal development and differentiation and in the function of the peripheral and central nervous system [6].

\section{NTRK fusions \& carcinogenesis}

NTRK fusions are the most common oncogenic mechanism in NTRK-altered tumors. Typically, the $3^{\prime}$ region of NTRK1, NTRK2 or NTRK3 that include the kinase domain is joined with the $5^{\prime}$ region of a different gene forming hybrid genes due to intrachromosomal or interchromosomal rearrangements. This hybrid gene encodes for a chimeric fusion oncoprotein responsible of ligand-independent constitutional activation of the TRK pathways [7]. It has been demonstrated in preclinical models that fusion oncoprotein may contribute to partial or complete loss of the immunoglobulin-like domain of TRK which has an inhibitory control of the signaling pathways in the absence of specific ligands [8]. NTRK fusions have been initially identified in colorectal and papillary thyroid carcinomas. 
Then, it has been found in several other types of adults and pediatric patients. The frequency of NTRK fusions in solid cancers remains unclear. The annual incidence of NTRK fusion-positive tumors is estimated to be 15005000 cancers in the USA [9]. Currently, we distinguish two major categories of cancers harboring NTRK fusions according to the frequency of the rearrangement. The first category comprised rare tumor types associated with high frequency of NTRK fusions such as infantile fibrosarcoma, secretory breast carcinoma, congenital mesoblastic nephroma, mammary analog secretory carcinoma in which NTRK fusions can be found in more than $90 \%$ of cases. The second group comprised more common solid tumors with lower frequencies of NTRK fusions such as breast, colorectal and lung cancers [5].

\section{Detection of NTRK fusions}

DNA-based next-generation sequencing (NGS) was predominantly used and has been effective in the clinical detection of NTRK rearrangements [10]. However, not all assays detect certain NTRK fusions, especially NTRK2 and NTRK3 rearrangements. The addition of targeted RNA sequencing, as a complementary method to NGS, has been associated with a sensitivity rate of $93 \%$ and a specificity rate of $100 \%$ [11]. FISH and RT-PCR could be an alternative to DNA-NGS, especially for cancer subtypes with high prevalence of NTRK rearrangements. The inconvenience of these methods is that it cannot detect more than one driver alteration at a time [12]. NTRK rearrangements could also be detected using plasma-based cell-free DNA [13].

\section{Available data on TRK inhibitors Larotrectinib}

Larotrectinib is a potent, orally administered, highly selective pan-TRK inhibitor. Drilon et al. reported the first data concerning the efficacy and safety of patients with TRK fusion-positive cancer treated with larotrectinib. 55 patients were enrolled and the age ranged from 4 months to 76 years. 12 patients (22\%) had salivary gland tumor, 11 patients (20\%) had soft tissue sarcomas, seven patients (13\%) had infantile fibrosarcoma and five patients (9\%) had thyroid tumor. The remaining patients had colon cancer, lung cancer, melanoma, GIST, cholangiocarcinoma, appendix tumor, breast cancer and pancreatic tumor. The overall response rate (ORR) was 75\% (95\% CI, 61-85\%). The median time to response was 1.8 months. The median progression-free survival (PFS) and duration of response were not reached after a median follow-up of 9.9 months. At 1 year, $55 \%$ of patients remained progression-free. No adverse event of grade 3 or higher which was considered to be related to larotrectinib was observed in more than $5 \%$ of patients. No treatment discontinuation owing to drug-related adverse events has occurred [10].

Moreover, Laetsch et al. reported the results of a multicenter, open-label, Phase I-II study enrolling infants, children and adolescents of 1 month to 21 years, with locally advanced or metastatic solid tumors or central nervous system tumors that have relapsed, progressed or were refractory to available therapies regardless of TRK fusion status and treated with larotrectinib. 24 patients were enrolled, 17 of them had tumors harboring TRK fusions. The median age of patients was 4.5 years. In the group of TRK fusion-positive cancers, 15 patients (88\%) had sarcomas (eight had infantile fibrosarcoma and seven had other types of soft tissue sarcomas) and two patients (12\%) had papillary thyroid cancer. The recommended dose of larotrectinib for Phase II study was $100 \mathrm{mg} / \mathrm{m}^{2}$ twice daily (maximum $100 \mathrm{mg}$ per dose). The ORR was $93 \%$ (14/15) in the group of patients with TRK fusionpositive cancer. No objective response to larotrecinib was reported in the TRK fusion-negative cancers. The most frequent adverse events of all grades were increased aspartate and alanine aminotransferase (42\%) followed by leucopenia $(21 \%)$, decrease neutrophil count $(21 \%)$ and vomiting $(21 \%)$. In this trial, there was no grade 4 or grade 5 treatment-related adverse events [12].

More recently, Dubois et al. reported the efficacy of larotrectinib as neoadjuvant treatment in five patients with locally advanced TRK fusion-positive sarcomas and included in the Phase I-II study published in Lancet Oncology. Three patients had infantile fibrosarcoma and the two others had soft tissue sarcoma. Four patients had refractory disease to standard chemotherapy. All patients presented partial response according to RECIST 1.1 criteria and underwent surgical resection, three of them had R0 margins, one had R1 margins and the last patient had R2 margins. Three patients presented complete or near-complete pathological response to larotrectinib [14]. In November 2018, the FDA granted an accelerated approval to larotrectinib for adults and pediatric patients with NTRK fusion-positive tumors that are metastatic or where surgical resection is likely to result in severe morbidity. 


\section{Entrectinib}

Entrectinib is a potent ATP-competitive pan-TRK, ALK and ROS1 tyrosine kinase inhibitor approved in the treatment of non-small-cell lung cancer with ALK or ROS1 rearrangements. Available data from the two Phase I trials ALKA 372-001 and STARTRK-1 showed an important activity of entrectinib in patients with TRK fusionpositive cancers. Four patients were identified: one with colorectal cancer, one with glioneural tumor, one with lung adenocarcinoma and one with mammary analog secretory carcinoma. The ORR was 100\% and the median PFS was not reached [13]. More recently, Demetri et al. presented at last ESMO 2018 data concerning the efficacy and safety of entrectinib in patients with NTRK fusion-positive cancers in a pooled analysis of STARTRK-2, STRATRK-1 and ALKA 372-001. 50 for patients were evaluated including patients with baseline central nervous system (CNS) metastases. After a median follow-up of 15.5 months, the ORR was $57.4 \%$ (95\% CI: 43.2-70.8\%). The median duration of response was 10.4 months, the median PFS was 11.2 months and the median overall survival (OS) was 20.9 months. The median PFS in patients without CNS metastases was 12.0 versus 7.7 months in patients with CNS metastases.

In conclusion, entrectinib and larotrectinib showed an important activity in patients with locally advanced NTRK fusion-positive solid tumors. For the first time, the FDA approved larotrectinib in the treatment of solid tumors with specific genetic alterations regardless of origin site. Several other agents are under investigation in this setting. Nevertheless, the activity of these agents is counterbalanced by the emergence of acquired resistance to these agents.

\section{Financial \& competing interests disclosure}

The authors have no relevant affiliations or financial involvement with any organization or entity with a financial interest in or financial conflict with the subject matter or materials discussed in the manuscript. This includes employment, consultancies, honoraria, stock ownership or options, expert testimony, grants or patents received or pending, or royalties.

No writing assistance was utilized in the production of this manuscript.

\section{References}

1. Press Announcements - FDA approves first cancer treatment for any solid tumor with a specific genetic feature. www.fda.gov/newsevents/newsroom/pressannouncements/ucm560167.htm

2. FDA grants nivolumab accelerated approval for MSI-H or dMMR colorectal cancer. www.fda.gov/drugs/informationondrugs/approveddrugs/ucm 569366.htm

3. FDA approves larotrectinib for solid tumors with NTRK gene fusions. www.fda.gov/drugs/informationondrugs/approveddrugs/ucm626720.htm

4. Lemmon MA, Schlessinger J. Cell signaling by receptor tyrosine kinases. Cell. 141(7), 1117-1134 (2010).

5. Cocco E, Scaltriti M, Drilon A. NTRK fusion-positive cancers and TRK inhibitor therapy. Nat. Rev. Clin. Oncol. 15(12), 731-747 (2018).

6. Nakagawara A. Trk receptor tyrosine kinases: a bridge between cancer and neural development. Cancer Lett. 169(2), 107-114 (2001).

7. Vaishnavi A, Le AT, Doebele RC. TRKing down an old oncogene in a new era of targeted therapy. Cancer Discov. 5(1), 25-34 (2015).

8. Arevalo JC, Conde B, Hempstead BL, Chao MV, Martin-Zanca D, Perez P. TrkA immunoglobulin-like ligand binding domains inhibit spontaneous activation of the receptor. Mol. Cell. Biol. 20(16), 5908-5916 (2000).

9. Kheder ES, Hong DS. Emerging targeted therapy for tumors with NTRK fusion proteins. Clin. Cancer Res. Off. J. Am. Assoc. Cancer Res. 24(23), 5807-5814 (2018).

10. Drilon A, Laetsch TW, Kummar S et al. Efficacy of larotrectinib in TRK fusion-positive cancers in adults and children. $N$. Engl. J. Med. 378(8), 731-739 (2018).

11. Reeser JW, Martin D, Miya J et al. Validation of a targeted RNA sequencing assay for kinase fusion detection in solid tumors. J. Mol. Diagn. JMD. 19(5), 682-696 (2017).

12. Laetsch TW, DuBois SG, Mascarenhas L et al. Larotrectinib for paediatric solid tumours harbouring NTRK gene fusions: Phase 1 results from a multicentre, open-label, Phase I/I study. Lancet Oncol. 19(5), 705-714 (2018).

13. Drilon A, Siena S, Ou S-HI et al. Safety and antitumor activity of the multitargeted Pan-TRK, ROS1, and ALK inhibitor entrectinib: combined results from two Phase I trials (ALKA-372-001 and STARTRK-1). Cancer Discov. 7(4), 400-409 (2017).

14. DuBois SG, Laetsch TW, Federman N et al. The use of neoadjuvant larotrectinib in the management of children with locally advanced TRK fusion sarcomas. Cancer 124(21), 4241-4247 (2018). 